\title{
Simplified model for predicting impulsive loads on submerged structures to account for fluid-structure interaction
}

\author{
Timon Rabczuk, Esteban Samaniego, Ted Belytschko \\ Department of Mechanical Engineering, Northwestern University, 2145 Sheridan \\ Road, Room A212, Evanston, IL 60208-3111, USA
}

\begin{abstract}
A simplified method for accounting for the effects of fluid structure interaction (FSI) in sandwich structures subjected to dynamic underwater loads is developed. The method provides quite accurate predictions of the impulse on submerged structures for a large range of loads and core yield strengths. It is a simple model with two lumped masses, one of which is subjected to an incident wave and a rheological model to represent the core. It enables phenomena such as buckling of the components of the core to be taken into account and is simple enough to be used as a design tool. Comparisons with calculations of complete fluid-structure models show very good agreement.
\end{abstract}

Key words: sandwich structures, reduced model, buckling, fluid-structure interaction

\section{INTRODUCTION}

Sandwich structures have substantial potential for many applications requiring light weight and high strength. Deshpande and Fleck [2, 3, 4], Deshpande et al. [5], Maekinen [9], Qiu et al. [10] and Xue and Hutchinson [13, 16, 17, 18] have studied the behavior and properties of sandwich shells. In References $[14,16]$, the dynamic response of sandwich shells to impulsive loads was considered. It was shown that substantial benefits accrue to sandwich shells in underwater response to impulsive loads. However, the fluid-structure interaction models in the studies were substantially simplified models. For example, in $[16,17]$, fluidstructure interaction effects were accounted for by a so-called Taylor model

Email address: t-belytschko@northwestern.edu (Ted Belytschko). 
which is based on the one-dimensional model of a wave interacting with a mass equal to that of the outside plate at one end [12]. The impulse on the mass, which is substantially lower than the impulse of the initial wave, was then applied to a complex model of the sandwich structure.

However, the single mass model substantially underestimates the impulse to which the sandwich is subjected as shown in section 3. Furthermore, the analysis of sandwich plates under large dynamic loads in underwater environments by complete finite element models that account fully for fluid-structure interaction effects is quite time consuming because the sandwich structure model requires high resolution to capture buckling modes and the fluid model requires high resolution to capture impulsive waves. Thus, complete models for fluid-structure interaction are very large and not suitable for design studies.

In a previous work [11], the authors presented a homogenized model for the core of sandwich beams for the purpose of simplifying coupled fluid-structure analysis of sandwich shells. It was shown that the agreement between the homogenized model and the fully detailed one was quite good. Significant computational savings were achieved by this model with respect to a complete analysis. The present contribution can be seen as an alternative for reducing computational complexity. Here, the fluid part of the model is simplified to provide loads on the structure that reflect the effects of fluid-structure interaction. It is intended as a computational tool for the design process.

In this method, the impulse imparted to the face sheet of the sandwich structure facing the fluid is predicted by a two degree of freedom model with a core representation. The methodology employs a simple model of the core similar to that in Rabczuk et al. [11]. As in Xue and Hutchinson [14, 15], the load on the outside plate obtained by this simplified method is considered the "fluidstructure" corrected load. This load is then applied to an uncoupled model of the structure to obtain estimates of its deformation in water. By enabling an uncoupled analysis of the structure, it provides significant savings in design and analysis.

\section{Formulation}

To determine the load imparted by the fluid, the sandwich structure is modelled as a system of two masses, $m_{1}$ and $m_{2}$, connected by a rheological model. The mass of the core is lumped at the upper and lower sandwich plate. This simplification seemed to be reasonable since wave propagation effects through the core play a minor role as shown later. A plot of a typical law governing the constitutive behavior of the rheological model can be seen in Fig (1). This law is obtained by a separate finite element buckling analysis of the core and 

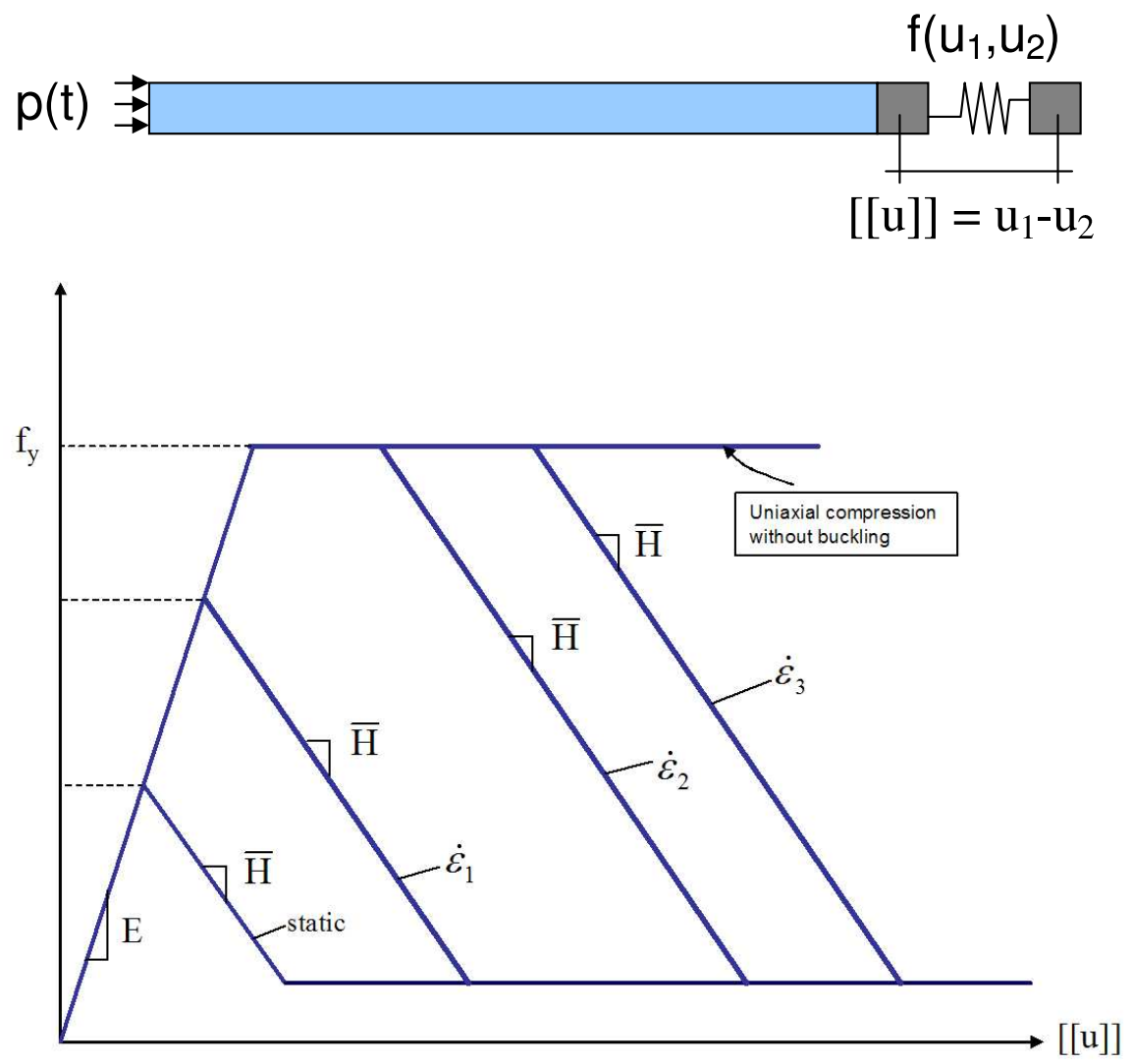

Fig. 1. 1D rheological model for core

depends on the material and structural characteristics of the core as suggested in Rabczuk et al. [11]. In the figure, $[[u]]$ denotes the difference between the displacement of the face sheet in contact with the water, $u_{1}$, and the displacement of the other face sheet, $u_{2}$. The softening branch of the curve is intended to capture the structural behavior of the core after buckling of its elements occurs. One difficulty is that the buckling curves depend on the load rate. Fast loads, which we are interested in, lead to a substantial increase in the peak strength. Typical buckling curves for different load velocities are illustrated in figure 1. In Rabczuk et al. [11], buckling curves depending on the strain rate were introduced. We will adopt this concept for our model.

We use a one-dimensional description of the fluid. Its action over the structure (see Fig. (2)) is modelled as an impedance element which models the fluidstructure interaction (a dashpot) plus the pressure produced by the incoming wave. We will be concerned with loads of the form

$$
p(t)=p_{0} e^{-t / t_{0}}
$$

where $p$ is the current pressure, $p_{0}$ is the applied pressure at time $t=0$, acting in $m_{1}$, the mass modelling the face sheet that is in contact with the fluid. 
The model shown in Fig (2) is developed as follows. Consider the momentum balance for $m_{1}$ :

$$
-m_{1} \ddot{u}_{1}-f\left(u_{1}, u_{2}\right)=\rho_{w} c_{w}^{2} \partial_{x} u_{w}(\cdot, 0)
$$

where $\rho_{w}$ and $c_{w}$ are, respectively, the density and the wave speed of the fluid, and $u_{w}(t, x)$ is the displacement field in the fluid. We assume that the one-dimensional wave equation governs the behavior of the fluid, so:

$$
\partial_{x}^{2} u_{w}=c_{w}^{2} \ddot{u}_{w}
$$

where

$$
c_{w}^{2}=\frac{K}{\varrho}
$$

where $K$ is the bulk modulus.

The d'Alembert solution of Eq. (3) has the form

$$
u_{w}(x, t)=g(c t-x)+h(c t+x)
$$

Adding the term $2 \rho_{w} c_{w}^{2} g^{\prime}$ to both sides of Eq. (2), we obtain

$$
-m_{1} \ddot{u}_{1}-f\left(u_{1}, u_{2}\right)+2 \rho_{w} c_{w}^{2} g^{\prime}=\rho_{w} c_{w}^{2} \partial_{x} u_{w}(\cdot, 0)+2 \rho_{w} c_{w}^{2} g^{\prime}
$$

Substituting Eq.(5) into Eq. (6), we have that

$$
\rho_{w} c_{w}^{2} \partial_{x} u_{w}+2 \rho_{w} c_{w}^{2} g^{\prime}=\rho_{w} c^{2}\left(g^{\prime}+h^{\prime}\right)=\rho_{w} c_{w} \dot{u}_{w}
$$

Bearing in mind that

$$
\dot{u}_{w}(t, 0)=\dot{u}_{1}(t)
$$

and that

$$
2 p(t)=2 \rho_{w} c_{w}^{2} g^{\prime}
$$

if Eq. (7) is substituted into Eq. (6), it yields

$$
m_{1} \ddot{u}_{1}+\rho_{w} c_{w} \dot{u}_{1}+f\left(u_{1}, u_{2}\right)-2 p_{0} e^{-t / t_{0}}=0
$$



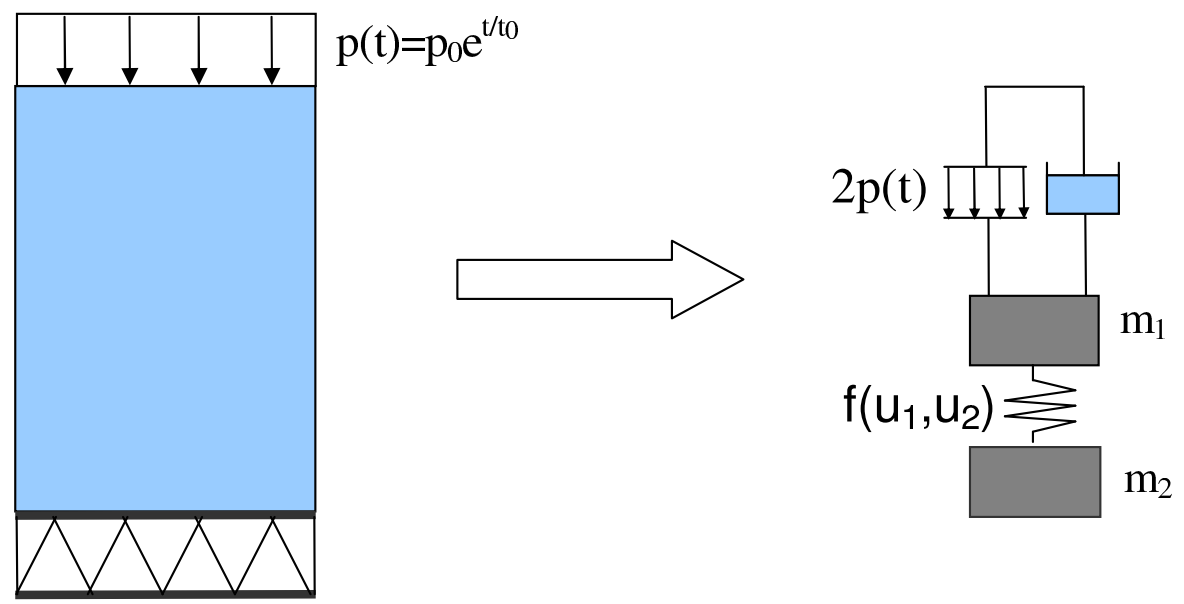

Fig. 2. The complete fluid-structure system and its two-degree-of-freedom representation

We also have that

$$
m_{2} \ddot{u}_{2}-f\left(u_{1}, u_{2}\right)=0
$$

Eqs. (10) and (11) state the momentum balance in each of the masses of the proposed rheological model.

Let us now define

$$
\begin{aligned}
& \boldsymbol{M}:=\operatorname{diag}\left(m_{1}, m_{2}\right) \\
& \boldsymbol{C}:=\operatorname{diag}\left(\rho_{w} c_{w}, 0\right) \\
& \boldsymbol{P}(t):=\left[2 p_{0} e^{-t / t_{0}}, 0\right]^{T} \\
& \boldsymbol{U}:=\left[u_{1}, u_{2}\right]^{T} \\
& \boldsymbol{F}(\boldsymbol{U}):=\left[f\left(u_{1}, u_{2}\right),-f\left(u_{1}, u_{2}\right)\right]^{T}
\end{aligned}
$$

Then equations (10) and (11) can be written in compact form as

$$
\boldsymbol{M} \ddot{\boldsymbol{U}}+\boldsymbol{C} \dot{\boldsymbol{U}}+\boldsymbol{F}(\boldsymbol{U})-\boldsymbol{P}(t)=\mathbf{0}
$$

It is important to mention that Eq. (12) is only valid as long as no cavitation takes place at any point of the fluid. The above is a system of two ordinary differential equations in two unknowns and it can easily be handled by a standard package such as MATLAB. For the impulsive loads considered here, it can be solved in less than a second on any PC. The central difference method as explained e.g. in Belytschko et al. [1] can also be used. The effect of cavitation on the impulse is obtained by the correction of Deshpande and 
Fleck [4].

\section{$3 \quad$ Numerical results}

\subsection{Core with hardening}

As mentioned in the Introduction, in the design of sandwich beams, it is crucial to know the total impulse that has been imparted to the structure. This impulse does not depend only on the wave in the fluid but also on the interaction of the fluid with the structure. In the examples presented in this section we will show how the proposed model can provide a good estimate of this impulse. Comparisons with more detailed models and with the reduced model of Deshpande and Fleck [4] will be also presented. In the following, we will call our simplified model a rheological model.

We consider a sandwich structure subjected to a far field impulse

$$
I_{0}=\int_{0}^{\infty} p_{0} e^{-t / t_{0}} d t=p_{0} t_{0}
$$

with the relaxation time $t_{0}=0.1 \mathrm{~ms}$ and the peak pressure $p_{0}=100 \mathrm{MPa}$ and hence $I_{0}=10 \mathrm{MPas}$. The height of the sandwich structure is $100 \mathrm{~mm}$ and the thickness of the lower and upper plate is $10 \mathrm{~mm}$. The core of the sandwich structure is a foam with density $\varrho_{0}=800 \mathrm{~kg} / \mathrm{m}^{3}$, Young's modulus $E=210 G P a$, Poisson's ratio $\nu=0.0$ and yield strength $f_{y}$ which varies as shown in Figure 3. The face sheets are assumed to be elastic with density of $8000 \mathrm{~kg} / \mathrm{m}^{3}$, Young's modulus $E=210 G P a$ and Poisson's ratio $\nu=0.0$. The normalized mass $\bar{m}$ (mass per area) of the sandwich structure is $0.24 \mathrm{~kg} / \mathrm{m}$ which is lumped to the lower and upper masses of our spring-mass-damper model. This data was adopted from Deshpande and Fleck [4] who developed an analytical model to approximate impulses of sandwich structures subjected to underwater loads based on cores with elastic, ideal-plastic material behavior. Note that in this example, we have no softening in the rheological model in accordance with Deshpande and Fleck [4].

Figure 3 shows the non-dimensional impulse $I / I_{0}$ versus the normalized yield strength $f_{y} / p_{0}$, where $I$ is the impulse subjected to the entire sandwich structure. The impulse includes cavitation corrections as described in Deshpande and Fleck [4]. The results of the simplified model are compared to a onedimensional finite element model of the fluid-structure system. The results of our model, the finite element analysis and the simplified model of Deshpande 


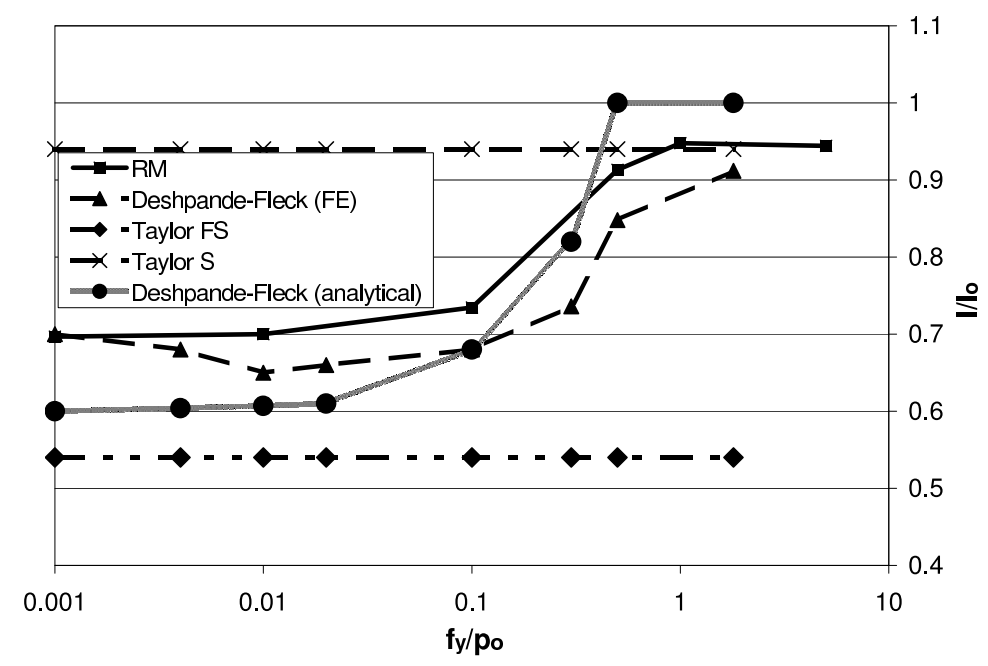

Fig. 3. Non-dimensional impulse for different yield strengths for a foam with elastic, ideal-plastic material behavior; $R M=$ our rheological model; Taylor F $S=$ impulse according to the Taylor analysis if the face sheet is assumed to be free standing; Taylor $S=$ impulse according to the Taylor analysis if the entire sandwich strcuture is assumed to be free standing; Deshpande and Fleck (FE): coupled calculation by finite elements with FSI in [4]; Deshpande and Fleck (analytical): an analytical model with wave propagation in core, see [4]

and Fleck [4] are shown in figure 3.

We were able to reproduce the impulses of the finite element analysis well. For non-dimensional yield strengths smaller than 0.01, no increase in the nondimensional impulse can be observed. Beyond this point, the non-dimensional impulse increases until a normalized yield strength of approximately 1 . After this, the non-dimensional impulse stays approximately constant at a level of 0.94 which is predicted well by our simplified model. The impulse from the Taylor analysis (see also Xue and Hutchinson [14]) is given in the figure too. The lower value of 0.54 is the impulse if the front face sheet of the sandwich structure is assumed to be the free-standing mass while the higher value of 0.94 is the non-dimensional impulse if the entire sandwich structure is assumed to be free-standing. Note, that the original results of Deshpande and Fleck [4] are shown for an impulse $I_{0}=20$ GPas. In their illustration $I_{0}$ is not the far field impulse subjected at the surface of the water but the impulse subjected to a rigid support.

\subsection{Core with Softening}

An important advantage of our model is that it can account for the effects of cores which exhibit strain softening. Strain softening is observed in the overall response of many truss-like or honeycomb structures when they buckle. 


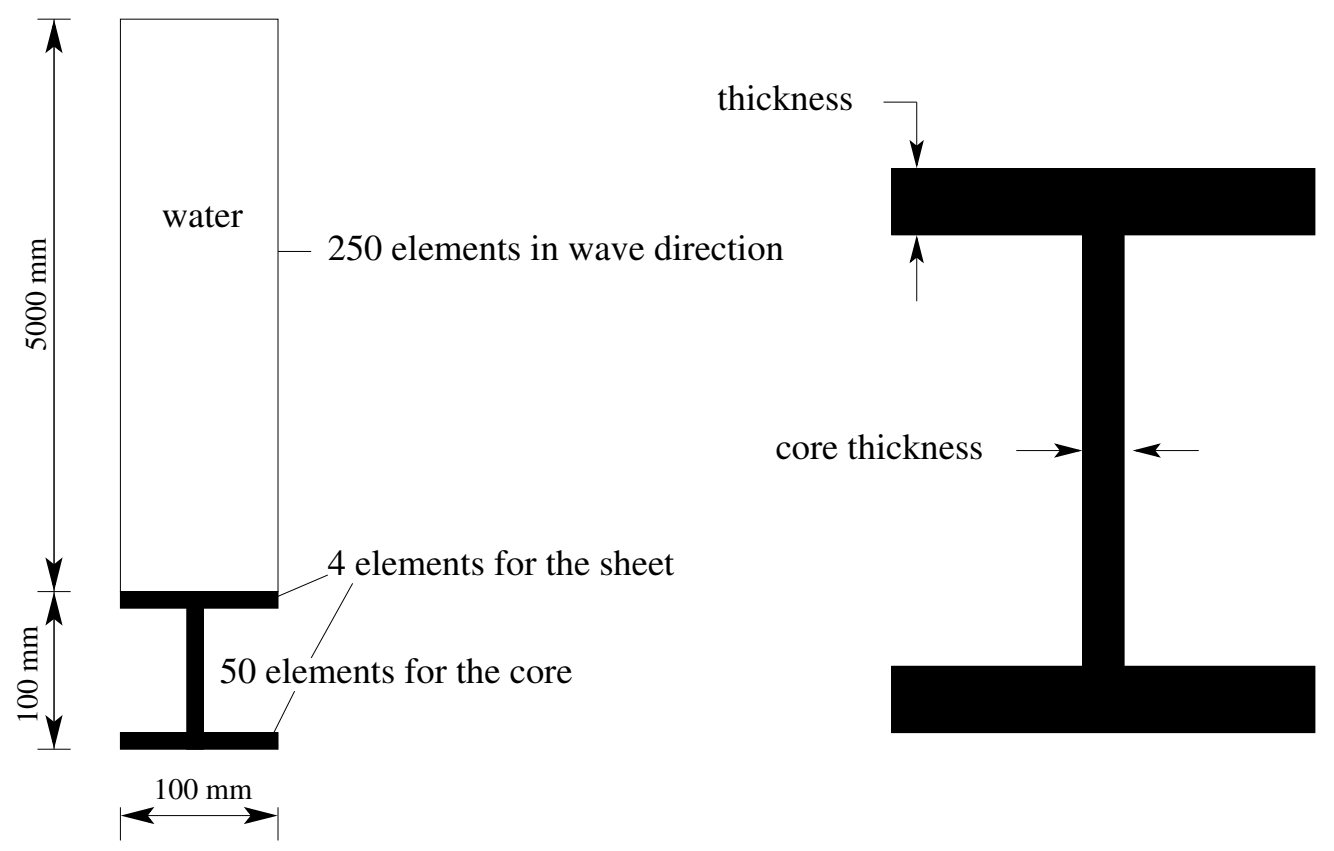

Fig. 4. Finite element model for sandwich plate; the model is a cross-section of the plate with a column of water through which the wave is propagated

Buckling results in a decrease of the force carried by the sandwich, which is manifested as overall strain softening in the core.

Consider a cross section of a sandwich plate as shown in figure 4 . The peak pressure, relaxation time, the far field impulse and the structural material (elastic, ideal-plastic) are the same as in the previous section. The material parameters for the sandwich structures are density $\varrho_{0}=8000 \mathrm{~kg} / \mathrm{m}^{3}$, Young's Modulus $E=210 G P a$ and Poisson's ratio $\nu=0.0$. The normalized mass $(\bar{m}=$ $\varrho_{0} L t$ with density $\varrho_{0}$, plate length and plate thickness $L$ and $t$, respectively) of the sandwich structure is $0.2 \mathrm{~kg} / \mathrm{m}$.

We use FEM analysis to obtain the response of the core. In the cases we considered, the basic unit of the core can be described by a beam. This beam is modelled by 50 beam elements. The response is then obtained for various prescribed velocities. B21 ABAQUS element model was used [7]. A small lateral force, $\sim 0.1 \%$ of the reaction force, was used to facilitate buckling. An example of load compression curves we obtained for vertical core elements with a length of $100 \mathrm{~mm}$ and a thickness of $1 \mathrm{~mm}$ is shown in Fig. 5 (this is a counterpart of Figure 1 for a specific core element).

The results of our simplified model will be compared with finite element solutions of the complete model in figure 4 obtained with the commercial finite element code ABAQUS [7]. The water is modelled via a linear equation of 


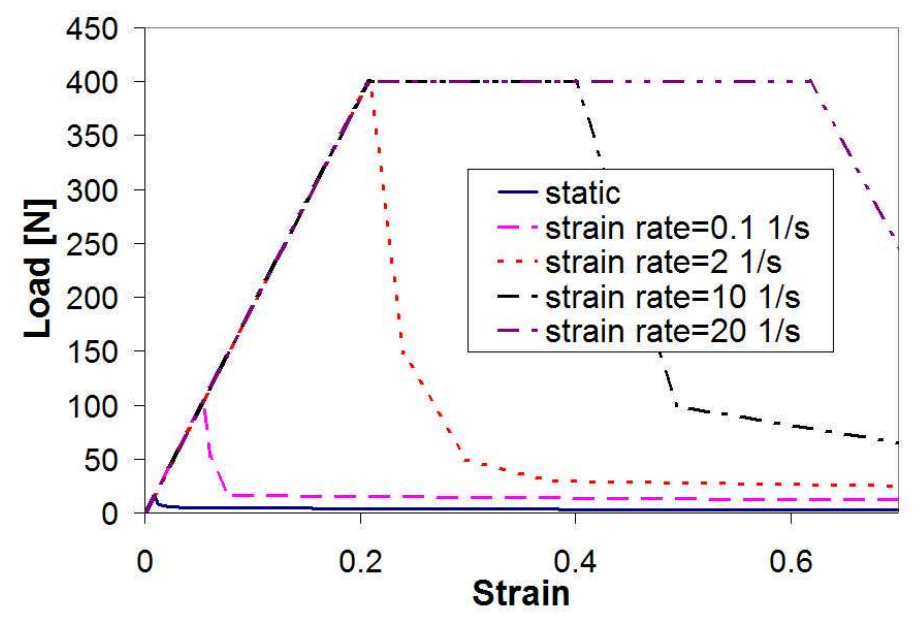

Fig. 5. Characterization of a vertical core for a beam of length $100 \mathrm{~mm}$ and thickness $1 \mathrm{~mm}$

state (EOS):

$$
p=\varrho_{0} c_{0}^{2}\left(1-\frac{\varrho_{0}}{\varrho}\right)
$$

with initial sound speed $c_{0}=1445 \mathrm{~m} / \mathrm{s}$ and inital density $\varrho_{0}=1000 \mathrm{~kg} / \mathrm{m}^{3}$. Cavitation is modelled by setting the pressure in the water to zero when it goes into tension. The fluid is assumed to be compressible and inviscid. The sandwich is discretized with beam elements. Reduced integration continuum elements (CPE4R) with consistent stabilization, Flanagan and Belytschko [6], are used for the water, see figure 4 . To obtain buckling of the core, a small perturbation is added to the system to break symmetry, i.e. lateral force is applied in the middle of the core.

In addition to the effective yield strength $f_{y}^{F E}$ ( $F E$ denotes the yield strength of the beam material in the finite element analysis), we also vary the volume fractions $V_{\text {core }} / V_{s w}$ of the structure, where $V_{\text {core }}$ is the volume of the steel in the core and $V_{s w}$ is the volume of the sandwich structure. We have varied the yield strength of the finite element model $f_{y}^{F E}$ from $2 M P a$ to $100 G P a$. This is an extremely large range, but we wanted to encompass a large range of materials from foams to dense metallic sandwiches as in Deshpande and Fleck [4]. The volume fraction $V_{\text {core }} / V_{s w}$ was varied from $2 \%$ to $8 \%$. The mass of the sandwich structure for different volume fractions is constant. Therefore, thicker beams in the core require thinner face sheets. Table 1 gives the data for the specimens we studied.

Before discussing the results, we would like to clarify some aspects of Table 1 . The strength $f_{y}^{R M}$ of our rheological model for different volume fractions, see for example the $8.5 \mathrm{~mm}$ thick face sheet with $f_{y}^{F E}=1 G P a$ and the $10.5 \mathrm{~mm}$ 
Table 1

Parameters of the spring-mass-damper model and the finite element model

\begin{tabular}{|c|c|c|c|c|c|}
\hline $\begin{array}{c}\text { thickness- } \\
\text { face sheets }\end{array}$ & core & $V_{\text {core }} / V_{\text {sw }}$ & $f_{y}^{F E}$ & $f_{y}^{R M}$ & $f_{y} / p_{0}$ \\
\hline $11.5 \mathrm{~mm}$ & $2 \mathrm{~mm}$ & 0.02 & $2 \mathrm{MPa}$ & $0.04 \mathrm{MPa}$ & 0.0004 \\
$11.5 \mathrm{~mm}$ & $2 \mathrm{~mm}$ & 0.02 & $10 \mathrm{MPa}$ & $0.2 \mathrm{MPa}$ & 0.002 \\
$11.5 \mathrm{~mm}$ & $2 \mathrm{~mm}$ & 0.02 & $100 \mathrm{MPa}$ & $2.0 \mathrm{MPa}$ & 0.02 \\
$11.5 \mathrm{~mm}$ & $2 \mathrm{~mm}$ & 0.02 & $200 \mathrm{MPa}$ & $4.0 \mathrm{MPa}$ & 0.04 \\
$11.5 \mathrm{~mm}$ & $2 \mathrm{~mm}$ & 0.02 & $1000 \mathrm{MPa}$ & $20.0 \mathrm{MPa}$ & 0.2 \\
$11.5 \mathrm{~mm}$ & $2 \mathrm{~mm}$ & 0.02 & $2000 \mathrm{MPa}$ & $40.0 \mathrm{MPa}$ & 0.4 \\
$11.5 \mathrm{~mm}$ & $2 \mathrm{~mm}$ & 0.02 & $10000 \mathrm{MPa}$ & $200.0 \mathrm{MPa}$ & 2.0 \\
$11.5 \mathrm{~mm}$ & $2 \mathrm{~mm}$ & 0.02 & $20000 \mathrm{MPa}$ & $400.0 \mathrm{MPa}$ & 4.0 \\
$11.5 \mathrm{~mm}$ & $2 \mathrm{~mm}$ & 0.02 & $100000 \mathrm{MPa}$ & $2000.0 \mathrm{MPa}$ & 20.0 \\
\hline $10.5 \mathrm{~mm}$ & $4 \mathrm{~mm}$ & 0.04 & $2 \mathrm{MPa}$ & $0.08 \mathrm{MPa}$ & 0.0008 \\
$10.5 \mathrm{~mm}$ & $4 \mathrm{~mm}$ & 0.04 & $10 \mathrm{MPa}$ & $0.4 \mathrm{MPa}$ & 0.004 \\
$10.5 \mathrm{~mm}$ & $4 \mathrm{~mm}$ & 0.04 & $100 \mathrm{MPa}$ & $4.0 \mathrm{MPa}$ & 0.04 \\
$10.5 \mathrm{~mm}$ & $4 \mathrm{~mm}$ & 0.04 & $200 \mathrm{MPa}$ & $8.0 \mathrm{MPa}$ & 0.08 \\
$10.5 \mathrm{~mm}$ & $4 \mathrm{~mm}$ & 0.04 & $1000 \mathrm{MPa}$ & $40.0 \mathrm{MPa}$ & 0.4 \\
$10.5 \mathrm{~mm}$ & $4 \mathrm{~mm}$ & 0.04 & $2000 \mathrm{MPa}$ & $80.0 \mathrm{MPa}$ & 0.8 \\
$10.5 \mathrm{~mm}$ & $4 \mathrm{~mm}$ & 0.04 & $10000 \mathrm{MPa}$ & $400.0 \mathrm{MPa}$ & 4.0 \\
$10.5 \mathrm{~mm}$ & $4 \mathrm{~mm}$ & 0.04 & $20000 \mathrm{MPa}$ & $800.0 \mathrm{MPa}$ & 8.0 \\
$10.5 \mathrm{~mm}$ & $4 \mathrm{~mm}$ & 0.04 & $100000 \mathrm{MPa}$ & $4000.0 \mathrm{MPa}$ & 40.0 \\
\hline $8.5 \mathrm{~mm}$ & $8 \mathrm{~mm}$ & 0.08 & $2 \mathrm{MPa}$ & $0.16 \mathrm{MPa}$ & 0.0016 \\
$8.5 \mathrm{~mm}$ & $8 \mathrm{~mm}$ & 0.08 & $10 \mathrm{MPa}$ & $0.8 \mathrm{MPa}$ & 0.008 \\
$8.5 \mathrm{~mm}$ & $8 \mathrm{~mm}$ & 0.08 & $100 \mathrm{MPa}$ & $8.0 \mathrm{MPa}$ & 0.08 \\
$8.5 \mathrm{~mm}$ & $8 \mathrm{~mm}$ & 0.08 & $200 \mathrm{MPa}$ & $16.0 \mathrm{MPa}$ & 0.16 \\
$8.5 \mathrm{~mm}$ & $8 \mathrm{~mm}$ & 0.08 & $1000 \mathrm{MPa}$ & $80.0 \mathrm{MPa}$ & 0.8 \\
$8.5 \mathrm{~mm}$ & $8 \mathrm{~mm}$ & 0.08 & $2000 \mathrm{MPa}$ & $160.0 \mathrm{MPa}$ & 1.6 \\
$8.5 \mathrm{~mm}$ & $8 \mathrm{~mm}$ & 0.08 & $10000 \mathrm{MPa}$ & $800.0 \mathrm{MPa}$ & 8.0 \\
\hline $8.5 \mathrm{~mm}$ & 0.08 & $20000 \mathrm{MPa}$ & $1600.0 \mathrm{MPa}$ & 16.0 \\
\hline $100000 \mathrm{MPa}$ & $8000.0 \mathrm{MPa}$ & 80.0 \\
\hline
\end{tabular}




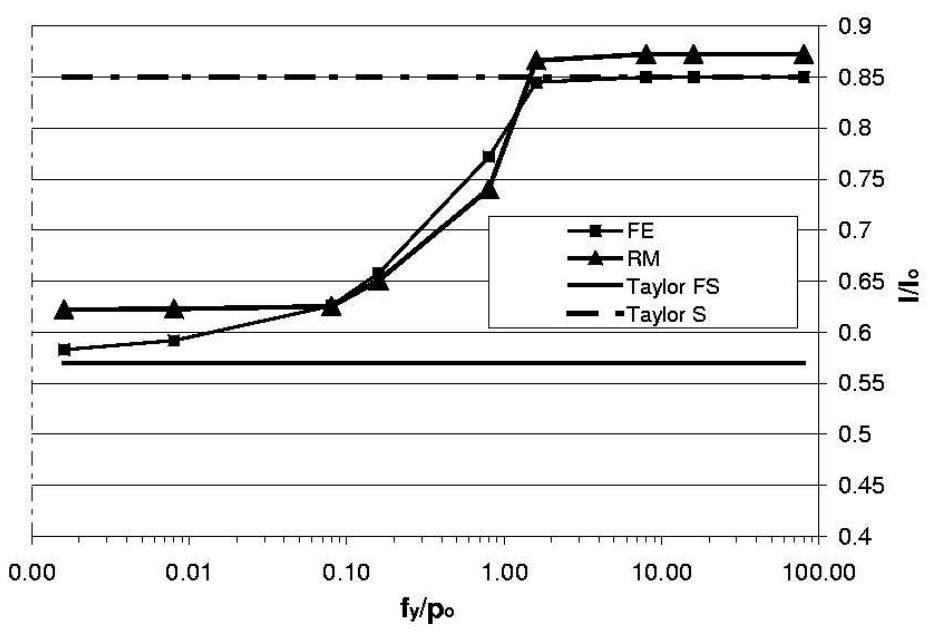

a) $2 \%$ volume fraction

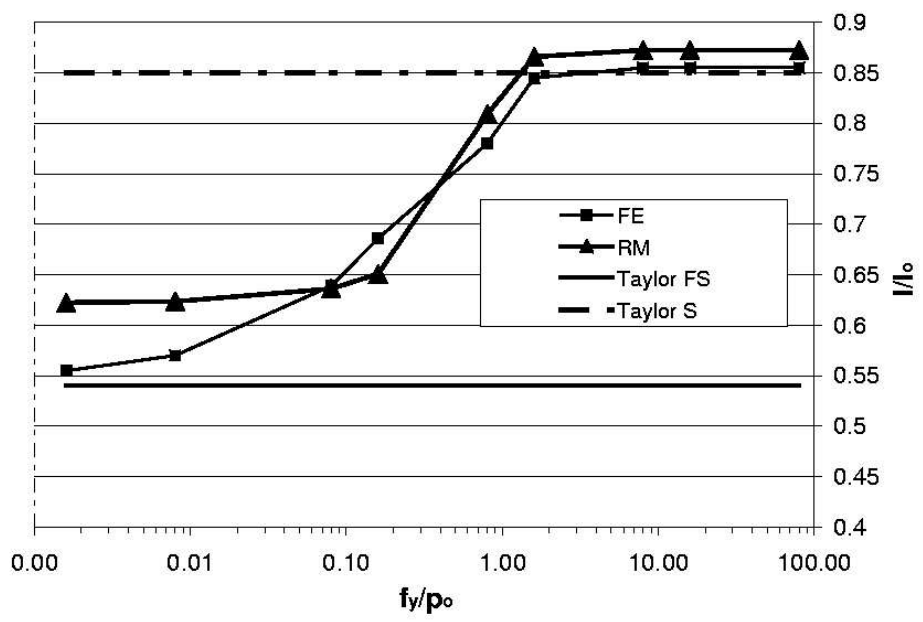

b) $4 \%$ volume fraction

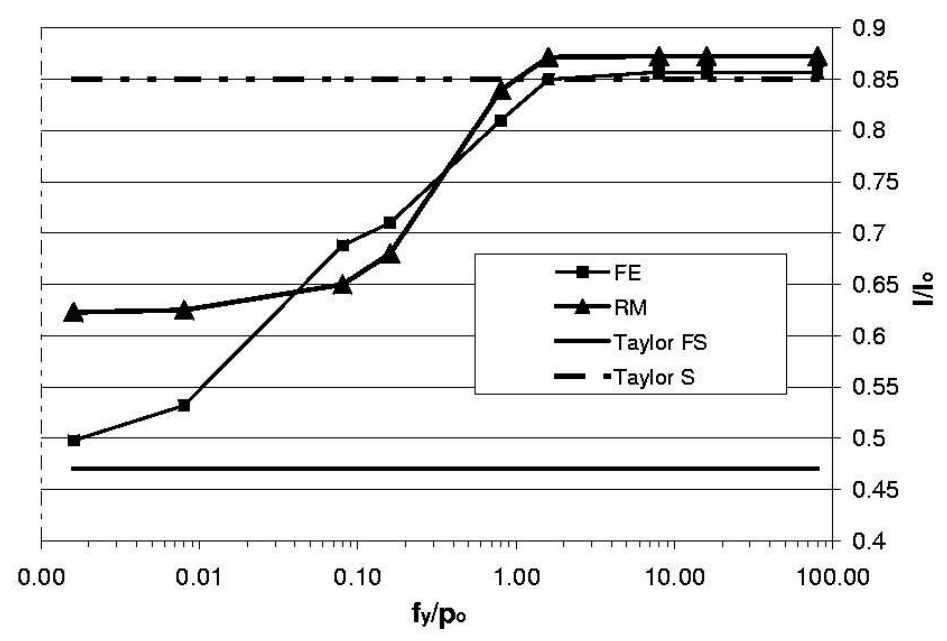

c) $8 \%$ volume fraction

Fig. 6. Non-dimensional impulse for different yield strengths for a truss core including buckling effects; $F E=$ complete fluid-structure finite element model results, $R M=$ our rheological model, Taylor FS = impulse according to the Taylor analysis if the face sheet is assumed to be free standing, Taylor $S=$ impulse according to the Taylor analysis if the entire sandwich 1 strcuture is assumed to be free standing 


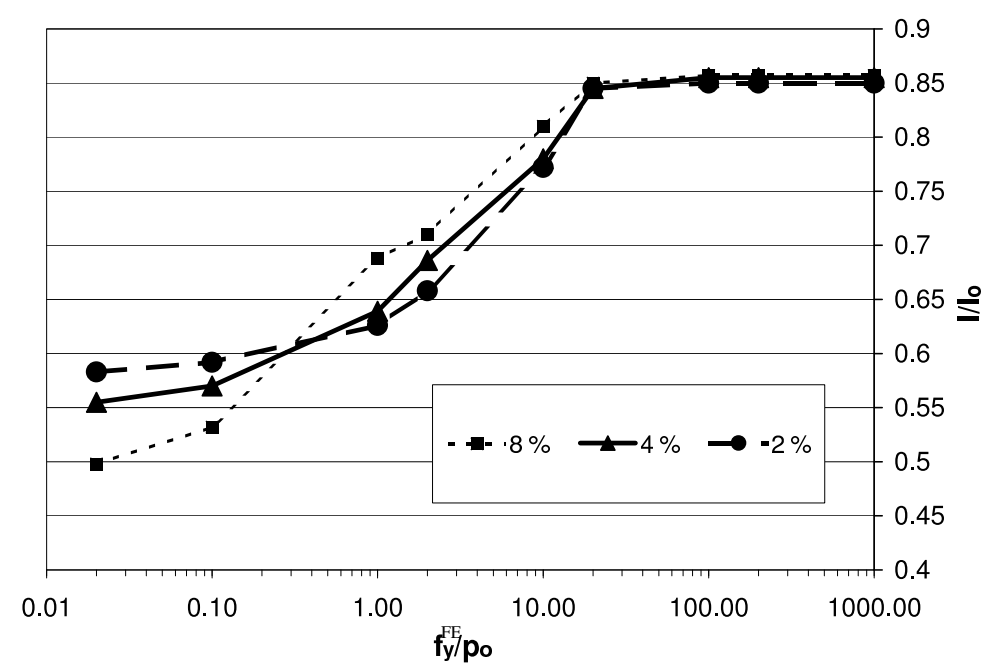

Fig. 7. Non-dimensional impulse for different yield strengths of the finite element analysis

thick face sheet with $f_{y}^{F E}=2 G P a$, are the same. We distinguish between the continuum yield stress $f_{y}^{F E}$ and the strength of our rheological model $f_{y}^{R M}$ as follows. The continuum yield stress is the yield stress of the elastic ideal-plastic constitutive model. The strength of our one dimensional rheological model is the strength obtained from a finite element analysis and includes buckling. $f_{y}^{R M}$ is obtained by dividing the maximum reaction force by the area of the face sheet (in our case it is only the thickness, since the depth of the model is unity).

Figure 6 shows the non-dimensional impulse versus the normalized strength $f_{y}^{R M} / p_{0}$. For low strengths, the non-dimensional impulse is nearly constant for all volume fractions we studied with our rheological model. The finite element model shows that $I / I_{0}$ decreases with increasing volume fraction for low strengths. This is due to the fact that the face sheet is thinner for higher volume fractions and therefore the applied impulse is smaller. The largest discrepancies between our rheological model and the finite element analysis occur for small stengths. However, strengths of about $f_{y}^{F E}=10 \mathrm{MPa}$ (this corresponds to a normalized strength $f_{y}^{R M} / p_{0}$ between 0.002 and 0.008 , see Table 1) are rather unrealistic, so we are not too concerned about this discrepancy.

At a normalized strength of about $f_{y}^{R M} / p_{0}=1$, no further increase in the applied impulse can be observed for the volume fractions we tested. The sandwich structure reacts rigidly, no buckling of the core is observed. We have included the impulses predicted by the Taylor analyses in figure 6 . As in the previous section, a lower impulse is obtained if the front face sheet is considered as free-standing than when the entire structure is considered to be free-standing. Our computations lie within these two bounds. We slightly overestimate the impulse for high strengths. 
In the most important part of the impulse range, the transition region between the lower and upper plateau, the rheological model is able to reproduce the applied impulses obtained by the finite element analysis. The error never exceeds $10 \%$. The errors are smallest for small volume fractions.

For cores with moderate strength, $0.1<f_{y}^{R M} / p_{0}<1$, and for the sandwich structures with small volume fraction, the impulse reduction due to fluidstructure interaction is greatest. This is not very clear in figure 6 since we used the strength of the rheological model as the abscissa. In figure $7, I / I_{0}$ is shown versus $f_{y}^{F E} / p_{0}$ to make clear the reduction in the impulse for lower volume fractions.

\subsection{Evaluation of the impulse estimates}

In this section, we will test the ability of our method to predict impulses applied to underwater sandwich structures in 2D. Consider the system as illustrated in figure 8. The pressure time history of eq. (1) with an impulse of $10 \mathrm{KPas}$ is applied on the top of the water model. This is the same impulse as we used in the previous section. The sandwich structure consists of two face sheets connected with vertical trusses. We have studied volume fractions of $2 \%, 4 \%$ and $8 \%$. Figure 8 also gives the impulse imparted to a solid plate. The material is elastic, ideal-plastic with a yield strength of $200 \mathrm{MPa}$, which corresponds to the yield strength for a stainless steel. We did not include hardening behavior in the constitutive model, first to be consistent with the previous section, and second to compare the results with other reduced models from the literature (Deshpande and Fleck [4] or Xue and Hutchinson $[14,15])$ which are designed for elastic, ideal-plastic constitutive models. The parameters for the structure are Young's modulus $E=210 G P a$, Poisson's ratio $\nu=0.0$ and density $\varrho_{0}=7800 \mathrm{~kg} / \mathrm{m}^{3}$. The parameters for the fluid are density $\varrho_{0}=1000 \mathrm{~kg} / \mathrm{m}^{3}$ and sound speed $c_{0}=1445 \mathrm{~m} / \mathrm{s}$. A linear equation of state (EOS) is used in the fluid.

The deformed sandwich structure at $15 \mathrm{~ms}$ for the different volume fractions is shown in figure 9 . While the core of the sandwich structure with a volume fraction of $8 \%$ behaves nearly rigidly, almost total collapse of the core can be observed for the $2 \%$ volume fraction sandwich structure.

Considering the imparted impulse to the sandwich, figure 10, it can be seen that the structures with lower volume fractions are subjected to less impulse. In other words, due to fluid-structure interaction, for lower volume fraction cores, a smaller part of the load is applied to the structure. We have illustrated the impulse in the middle of the sandwich structure since it is most comparable to our 1D model. Note, that the impulses vary over the length of 




Fig. 8. Fluid structure interaction model for sandwich structure under pressure wave loading

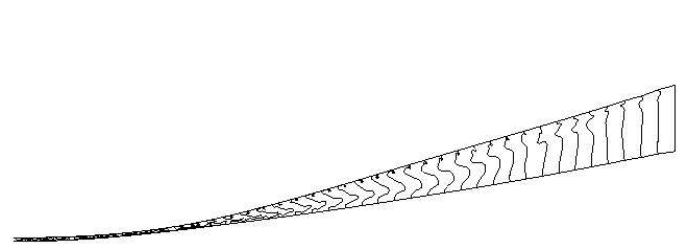

a) $2 \%$ volume fraction

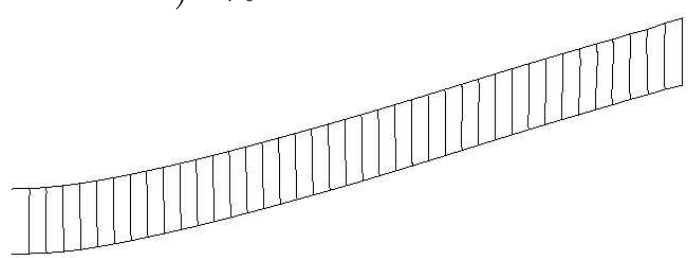

c) $8 \%$ volume fraction

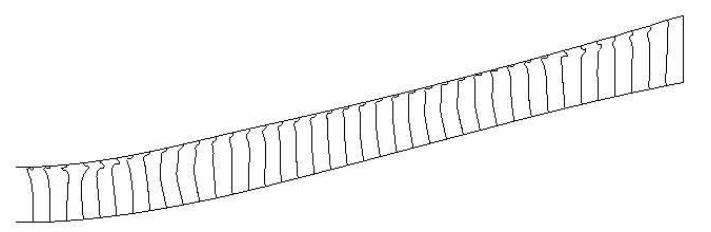

b) $4 \%$ volume fraction

Fig. 9. Deformed sandwich structure at $15 \mathrm{~ms}$ for different volume fractions 
the structure. The impulse increases with decreasing distance to the support. For the $8 \%$ structure, an impulse of $10.14 \mathrm{KPas}$ is imparted at the support, an impulse of $7.83 \mathrm{KPas}$ is imparted in the quarter points while the impulse in the middle is $7.19 \mathrm{KPas}$. The differences in the impulse over the length of the structure decrease with decreasing volume fraction. So, for the $2 \%$ volume fraction structure, the impulse above the support is $9.53 \mathrm{KPas}$, the impulse in the quarter point is $6.58 \mathrm{KPas}$ and the impulse in the middle is $6.43 \mathrm{KPas}$.

The impulse predicted by our model is also shown in figure 10. It can predict the imparted impulse of the FSI finite element analysis well; the best agreement is obtained for low volume fractions. The results of the Taylor analysis and the extended Taylor analysis proposed by Hutchinson and Xue [8] are shown in figure 10 as well. The Taylor analysis underestimates the imparted impulse. The extended Taylor analysis can capture the impulse for the low volume fractions pretty well. However, for large volume fractions, quite large discrepancies occur. Note, Hutchinson and Xue [8] introduce a 'core compression strength factor' $\lambda_{c}$ in their reduced model. They give values for different core types; the tetragonal truss core has a value of $\lambda_{c}=2 / 3$. The imparted impulses can differ significantly with the choice of $\lambda_{c}$. We have chosen $\lambda_{c}=1.0$ since the trusses of our core are straight. This is the value, Hutchinson and Xue [8] specifies for a square honeycomb which is one of the most resistant core types. The shear resistance factor which is also addressed in [8] is zero since our truss core does not have any shear resistance. Notice that in the Taylor and the extended Taylor analysis, the imparted impulse decreases with decreasing volume fraction, which is the opposite of the FSI finite element analysis. On the other hand, our rheological model reproduces this trend correctly.

To show the improvements in the imparted impulse for sandwich structures, the impulse of the solid plate is shown in figure 10 as well. As can be seen, a high volume fraction gives only slight improvement.

\subsection{Comparison of Displacements for Uncoupled and Coupled Models}

In the optimization of sandwich designs, the maximum displacements of a given sandwich structure and not the imparted impulse are of interest. Therefore, we performed full fluid-structure computations and computations where the impulse obtained from our rheological model is directly applied to the face sheet of the sandwich structure and compared the maximum midpoint displacement of the inside face sheet. We studied impulses between $7.5 \mathrm{KPas}$ and $15 K P A s$ with the time history of eq. (1). In addition, we also varied the core geometry. In addition to the vertical cross core, cores where the trusses are sloped with a 45 degree angle as shown in figure 11 are considered. The latter truss cores are of interest since they have shear resistance. As in the 


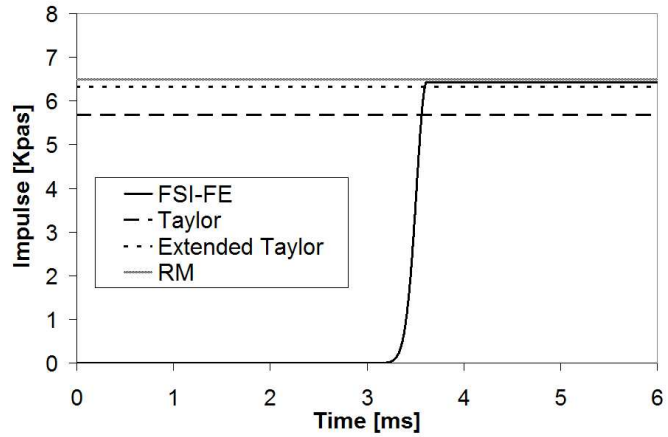

a) $2 \%$ volume fraction

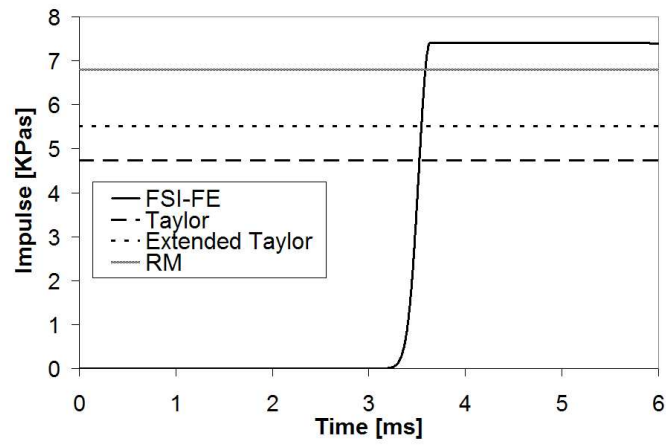

c) $8 \%$ volume fraction

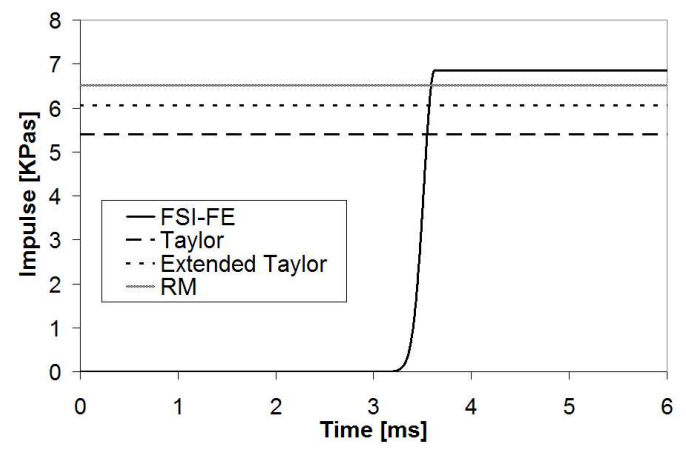

b) $4 \%$ volume fraction

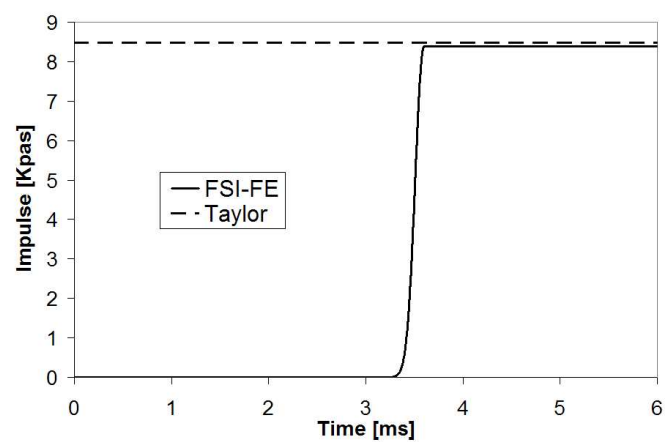

d) solid plate

Fig. 10. Time history of the impulse for the $2 \mathrm{D}$ analysis for a) to c) sandwich structures of different volume fractions, d) solid plate

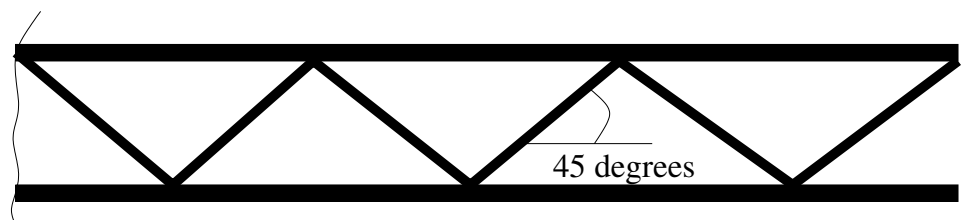

Fig. 11. Schematic of sandwich structure with diagonal truss core previous section, we study three different volume fractions, $2 \%, 4 \%$ and $8 \%$.

The results are shown in figure 13a for the vertical core and figure 13b for the diagonal core. In this figure, $u$ is the maximum displacement and $L$ is the half-length of the structure, $1 \mathrm{~m}$. The non-dimensional impulse is given by

$$
\bar{I}=\frac{I_{0}}{\bar{M} \sqrt{\sigma_{Y} / \varrho_{0}}}
$$

where $\bar{M}=\varrho_{0} L t$ is the mass per unit area, also called normalized area, with plate thickness $t, I_{0}$ is the far field impulse, $\sigma_{Y}$ the material yield strength and $\varrho_{0}$ is the density of the core.

We first note the superiority of the diagonal truss core over the vertical truss 
core which is due to its contribution to shear stiffness and strength. The agreement in the maximum midpoint displacement between the fluid-structure interaction computation and the uncoupled calculations is generally very good. In some cases, an increasing volume fraction and increasing impulse leads to a larger error, but not always. This might have several causes. First, we apply a uniform impulse over the entire length of the sandwich structure. However, the impulse is larger near the support due to the increased stiffness provided by the supports. This is shown in Fig. 12 which shows the impulse histories at three points for the fully coupled calculation. However, its effect appears to be moderate since the impulse near the supports do not contribute as much to the deflection. For example, for the straight core, the impulses (between the FSI and RM computations) for the $2 \%$ volume fraction agree better than for the $4 \%$ volume fraction, but the opposite is observed for the displacements, see figure 13a. Therefore, we conclude, that the variation of the impulse over the length has some influence.

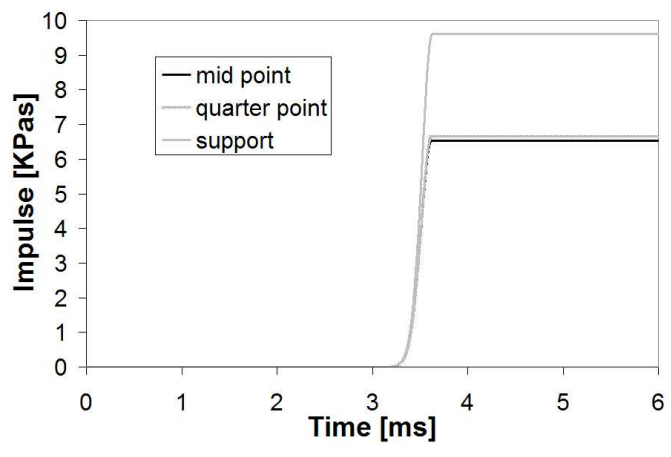

a)

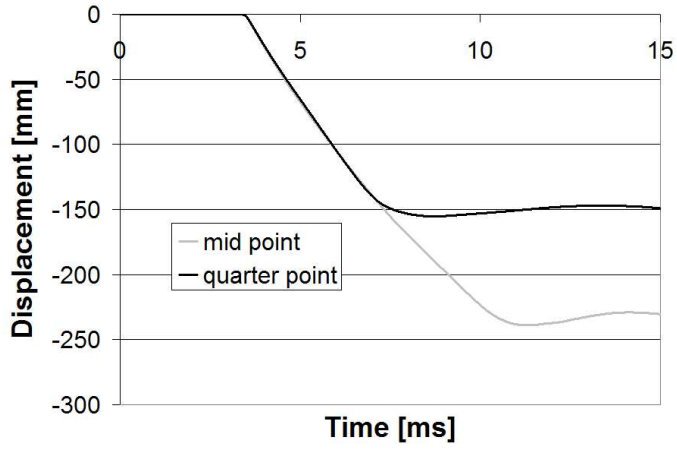

b)

Fig. 12. a) Impulse and b) displacement time history at different positions for the $2 \%$ sandwich structure

Second, we recall from the previous section that the error in impulse (between the FSI simulation and our rheological model) for the vertical truss core increases with increasing volume fraction. This tendency is also observed when the impulse is increased. The absolute value of the error increases though the percentage error is almost constant. However, for the diagonal truss core, the relative discrepancies in impulse between the FSI simulation and the rheological model are similar for all volume fractions. As can be seen, the largest discrepancies for the diagonal truss core are obtained with the $4 \%$ volume fraction. The discrepancies in the impulse are probably due to errors in the load-deflection curve of the rheological model which arise from the dynamic analysis on the core elements which are used to obtain them, because it is sometimes difficult to filter noise out of the load-deflection curves.

Some other comments on the issue of nonuniform impulses that are applied to the face sheet in the complete FSI analysis are in order. While for the vertical core, the impulses applied to the face sheet increase with decreasing distance 




a) straight truss core

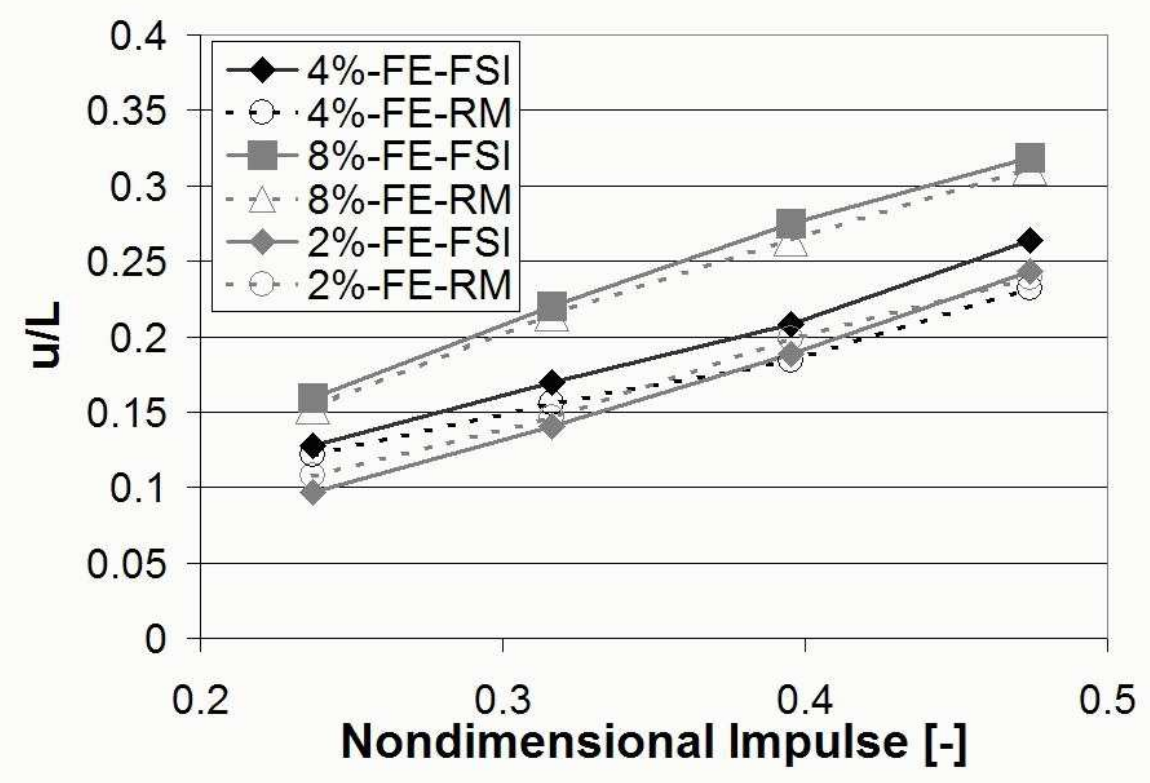

b) diagonal truss core

Fig. 13. Normalized maximum mid-displacement versus the non-dimensional impulse for different cores with different volume fractions; FE-RM is the finite element model of structures with loads from the rheological model; FE-FSI is the complete coupled fluid-structure computation; percentage gives volume fraction

to the support, the impulses for the diagonal truss core vary more over the length of the sandwich structure than for the vertical truss core. Especially for higher volume fractions, large deformations in the face sheets (since the face sheets are thinner) were also observed which causes two dimensional (wave reflection) effects which cannot be captured by our model. However, the results 
are remarkably good considering the simplicity of our model.

\section{Concluding Remarks}

We have presented a simplified model for estimating the impulsive load on a sandwich plate submerged in water due to a dynamic wave loading that accounts for fluid-structure interaction. The model consists of two masses joined by a core model that is based on the buckling strength and plastic yield strength of the core. The fluid-structure interaction effect is accounted for by the d'Alembert solution to the one-dimensional wave equation. As a result, the model only involves two ordinary differential equations in time, which can easily be treated by any standard package.

One drawback of the procedure is that it is necessary to develop a forcedeflection curve for the core. This force-deflection law must account for the buckling strength, its dependence on the rate of loading, and the yield strength of the material. The interesting feature of this study is that once a rheological model for the force-deflection behavior has been found for the core, then a two degree-of-freedom model as described here suffices for incorporating the fluidstructure interaction effects with reasonable accuracy. This model then enables one to probe the behavior of various designs over a large range of impulses and other variables by a detailed finite element model without explicitly accounting for fluid-structure interaction. Since the modelling of the fluid that surrounds a structure usually entails substantial computational expense, this approach can be quite beneficial in design studies.

The excellent agreement between the simplified model and the complete coupled finite-element model also offers some interesting insight into the mechanics of sandwich structures when subjected to impulsive underwater loads. It is clear that wave propagation through the core has little effect on the fluidstructure interaction effects, since the results for the simple model agree well with the fully coupled model. In other words, for a highly impulsive load, if the distance between the sandwich plates is sufficiently small $(10 \%$ or less of the span) so that the traversal time is much less than the response time of the structure, the load on the top plate depends primarily on the load-deflection behavior of the core. As can be seen from figure 12b, it takes more than $8 \mathrm{~ms}$ for the structure to reach its maximum displacement, whereas the travel time through the core is approximately $0.02 \mathrm{~ms}$ which is a ratio of 0.0025 . Although dynamic effects may change the force-deflection behavior due to strain-rate effects and the acceleration of buckling, the wave propagation effects through the core appear to be secondary. 


\section{Acknowledgement}

The support of Office of the Naval Research is gratefully acknowledged.

\section{References}

[1] T. Belytschko, W. K. Liu, and B. Moran. Nonlinear finite elements for continua and structures. John Wiley \& Sons Ltd., New York, 2000.

[2] V. S. Deshpande and N. A. Fleck. Isotropic constitutive models for metallic foams. Journal of the Mechanics and Physics of Solids, 48(6-7):12531283, 2000.

[3] V. S. Deshpande and N. A. Fleck. Collapse of truss core sandwich beams in 3-point bending. International Journal of Solids and Structures, 38: 6275-6305, 2001.

[4] V. S. Deshpande and N. A. Fleck. One-dimensional shock response of sandwich plates. submitted to Journal of the Mechanics and Physics of Solids, 2004.

[5] V. S. Deshpande, N. A. Fleck, and M. F. Ashby. Effective properties of the octet-truss lattic material. Journal of the Mechanics and Physics of Solids, 49:1747-1769, 2001.

[6] D.P. Flanagan and T. Belytschko. A uniform strain hexahedron and quadrilateral with ortogonal hourglass control. International Journal for Numerical Methods in Engineering, 17(5):679-706, 1981.

[7] ABAQUS/Explicit User's Manual Version 6.3. Hibbit, Karlsson and Sorensen Inc., 2002.

[8] J.W. Hutchinson and Z. Xue. Metal sandwich plates optimized for pressure impulses. Preprint, 2004.

[9] K. Maekinen. Underwater shock loaded sandwich structures. Doctoral Thesis, Department of Aeronautics, Royal Institut of Technology, Sweden, 1999.

[10] X. Qiu, V. S. Deshpande, and N. A. Fleck. Finite element analysis of the dynamic response of clamped sandwich beams subject to shock loading. European Journal of Mechanics A/Solids, 22:801-814, 2003.

[11] T. Rabczuk, J.Y. Kim, E. Samaniego, and T. Belytschko. Homogenization of sandwich structures. International Journal for Numerical Methods in Engineering, 61:1009-1027, 2004.

[12] G. I. Taylor. Aerodynamics and the Mechanics of Projectiles and Explosions, volume III of The Scientific Papers of Sir Geoffrey Ingram Taylor, chapter The pressure and impulse of submarine explosion waves on plates, pages 287-303. Cambridge University Press, 1963.

[13] Z. Xue and J.W. Hutchinson. Crush dynamics of square honeycomb 
sandwich cores. accepted in International Journal for Numerical Methods in Engineering.

[14] Z. Xue and J.W. Hutchinson. Preliminary assessment of sandwich plates subject to blast loads. International Journal of Mechanical Sciences, 45: 687-705, 2003.

[15] Z. Xue and J.W. Hutchinson. A comparative study of blast-resistant metal sandwich plates. International Journal of Impact Engineering, 30: 1283-1305, 2004.

[16] Z. Xue and J.W. Hutchinson. Constitutive model for metallic sandwich structures: I-theory. Preprint, 2004.

[17] Z. Xue and J.W. Hutchinson. Constitutive model for metallic sandwich structures: II-application. Preprint, 2004.

[18] Z. Xue and J.W. Hutchinson. Constitutive model for quasi-static deformation of metallic sandwich cores. International Journal for Numerical Methods in Engineering, 61(13):2205-2238, 2004. 


\section{List of Figures}

1 1D rheological model for core

2 The complete fluid-structure system and its two-degree-offreedom representation

3 Non-dimensional impulse for different yield strengths for a foam with elastic, ideal-plastic material behavior; $R M=$ our rheological model; Taylor $F S=$ impulse according to the Taylor analysis if the face sheet is assumed to be free standing; Taylor $S=$ impulse according to the Taylor analysis if the entire sandwich strcuture is assumed to be free standing; Deshpande and Fleck (FE): coupled calculation by finite elements with FSI in [4]; Deshpande and Fleck (analytical): an analytical model with wave propagation in core, see [4]

4 Finite element model for sandwich plate; the model is a cross-section of the plate with a column of water through which the wave is propagated

5 Characterization of a vertical core for a beam of length 100 $\mathrm{mm}$ and thickness $1 \mathrm{~mm}$

6 Non-dimensional impulse for different yield strengths for a truss core including buckling effects; $F E=$ complete fluid-structure finite element model results, $R M=$ our rheological model, Taylor $F S=$ impulse according to the Taylor analysis if the face sheet is assumed to be free standing, Taylor $S=$ impulse according to the Taylor analysis if the entire sandwich strcuture is assumed to be free standing

$7 \quad$ Non-dimensional impulse for different yield strengths of the finite element analysis

8 Fluid structure interaction model for sandwich structure under pressure wave loading

9 Deformed sandwich structure at $15 \mathrm{~ms}$ for different volume fractions

10 Time history of the impulse for the 2D analysis for a) to c) sandwich structures of different volume fractions, d) solid plate

11 Schematic of sandwich structure with diagonal truss core 
12 a) Impulse and b) displacement time history at different positions for the $2 \%$ sandwich structure

13 Normalized maximum mid-displacement versus the nondimensional impulse for different cores with different volume fractions; FE-RM is the finite element model of structures with loads from the rheological model; FE-FSI is the complete coupled fluid-structure computation; percentage gives volume fraction 